\title{
The Symmetry and Predictive Factors in Two-Stage Bilateral Hip Replacement Procedures
}

\author{
Krystian Kazubski ${ }^{1}$, Łukasz Tomczyk ${ }^{2}$ (D), Mariusz Ciszewski ${ }^{1}$, Jarosław Witkowski ${ }^{3}$, Paweł Reichert ${ }^{4}$ \\ and Piotr Morasiewicz $1, *$ (i) \\ 1 Department of Orthopaedic and Trauma Surgery, University Hospital in Opole, Institute of Medical Sciences, \\ University of Opole, Witosa 26, 45-401 Opole, Poland; krystian.kazubski@uni.opole.pl (K.K.); \\ mariusz.ciszewski@uni.opole.pl (M.C.) \\ 2 Department of Food Safety and Quality Management, Poznan University of Life Sciences, \\ Wojska Polskiego 28, 60-637 Poznan, Poland; tomczyk@up.poznan.pl \\ 3 Department of Sports Medicine, Wroclaw Medical University, Grunwaldzka 2, 50-355 Wrocław, Poland; \\ jaroslaw.witkowski@umed.wroc.pl \\ 4 Department of Trauma and Hand Surgery, Wroclaw Medical University, Borowska 213, \\ 50-556 Wroclaw, Poland; pawel.reichert@umed.wroc.pl \\ * Correspondence: piotr.morasiewicz@uni.opole.pl; Tel.: +48-77-45-20-624
}

Citation: Kazubski, K.; Tomczyk, Ł.; Ciszewski, M.; Witkowski, J.;

Reichert, P.; Morasiewicz, P. The

Symmetry and Predictive Factors in Two-Stage Bilateral Hip Replacement Procedures. Symmetry 2021, 13, 1472. https://doi.org/10.3390/sym13081472

Academic Editors: Jan Awrejcewicz and Alexander Shapovalov

Received: 20 July 2021

Accepted: 9 August 2021

Published: 11 August 2021

Publisher's Note: MDPI stays neutral with regard to jurisdictional claims in published maps and institutional affiliations.

Copyright: (C) 2021 by the authors Licensee MDPI, Basel, Switzerland. This article is an open access article distributed under the terms and conditions of the Creative Commons Attribution (CC BY) license (https:/ / creativecommons.org/licenses/by/ $4.0 /)$.

\begin{abstract}
Background: Approximately 10-25\% of total hip replacement patients undergo a bilateral procedure. The purpose of this study was to compare selected parameters associated with the first and second hip arthroplasty in patients undergoing two-stage treatment due to bilateral hip osteoarthritis and establish the predictive factors for the second procedure. Methods: This study compared the data on bilateral total hip replacement surgeries conducted in the period between 2017 and 2021 (42 patients). The following parameters from the first and second procedure were compared: the prosthetic stem, head, and insert cup size; type of cup insert; duration of anesthesia; duration of hospitalization; and the number of complications. Results: The mean duration of hospital stay at the time of the first total hip arthroplasty was 5.83 days and 5.4 days during the second stay. The mean stem sizes used during the first and second total hip replacement procedures were 7.11 and 7.09, respectively. The mean sizes of endoprosthetic cups used at the first and second total hip replacement procedures were 52.64 and 53.04, respectively. There were no significant differences between the mean prosthetic head size at the first and second surgery. The cup type used during the first and second surgery showed no difference. The mean duration of anesthesia used during the first and second total hip replacement surgery was $108.09 \mathrm{~min}$ and $104.52 \mathrm{~min}$, respectively. We recorded a mean of 0.07 complications per patient at the first surgery and 0.02 at the second surgery. Conclusions: Our study results showed symmetry duration of anesthesia, length of hospital stay, number of complications per patient, stem size, prosthetic head size, cup insert size, and cup insert type at the first and second surgery in patients with two-stage bilateral total hip arthroplasty. We observed a strong correlation between the stem sizes of the first and second hip endoprostheses. There was also a strong correlation between the cup sizes used during the first and second surgery.
\end{abstract}

Keywords: symmetry; predictive factors; two-stage; bilateral; hip replacement

\section{Introduction}

Total hip arthroplasty patients constitute a large proportion of orthopedic patients [1,2], with 1-2 million hip and knee replacement procedures performed annually in the US [1,2]. An estimated $2.34 \%$ and $4.55 \%$ of the US population over the age of 50 has had a total hip and total knee replacement surgery, respectively [1].

Bilateral total hip replacement has been recommended in patients with osteoarthritis of both hip joints [3-6]. Approximately $10-25 \%$ of total hip replacement patients undergo a bilateral procedure [3,7-9]. 
There are two management strategies in bilateral hip disease: a simultaneous (i.e., onestage) bilateral total hip arthroplasty or a two-stage procedure, with total hip arthroplasty of one hip at a time [3-11]. Although either strategy is favored by some orthopedic surgeons [3-11], most reports advocate the two-stage management, due to less severe complications, not as extensive procedures, less blood loss, more rapidly resumed daily functioning, and a shorter period of rehabilitation after arthroplasty on only one side $[3,6,8]$. The two-stage strategy may also be more beneficial for patients with bilateral osteoarthritis of the hip than the simultaneous bilateral arthroplasty, as the former affords an opportunity to assess the predictive factors for the second procedure [3,4].

The issue of assessing the symmetry of endoprosthetic parameters following bilateral hip replacement has not been fully investigated, and thus it has been scarcely reported [3-5]. Although the factors affecting the survivorship of bilateral hip prostheses have been evaluated $[3,4]$, the available literature contains no reports showing the predictive factors gleaned after the first arthroplasty that could affect the subsequent procedure involving the collateral hip joint. Learning the predictive factors for the second hip replacement surgery from the first procedure may facilitate the second procedure and lead to better outcomes $[3,4]$. Moreover, learning the effect of certain parameters of the first hip replacement, such as the size of the endoprosthesis, duration of the procedure, or the number of complications, may help plan the second-stage surgery and prepare the surgeon for possible challenges and complications, thus improving the treatment process [3].

We theorized that the parameters of the second-stage procedure would be symmetrical to those associated with the first total hip arthroplasty.

The purpose of this study was to compare selected parameters associated with the first and second hip arthroplasty in patients undergoing two-stage treatment due to bilateral hip osteoarthritis and establish the predictive factors for the second procedure.

\section{Material and Methods}

In the period from 2017 to 2021, a total of 47 patients underwent a two-stage bilateral total hip replacement at our clinic. The inclusion criteria for this study were: a history of two-stage bilateral total hip arthroplasty for the degenerative joint disease of the hips, and a complete set of medical and radiographic records. Study exclusion criteria included a two-stage bilateral total hip arthroplasty due to femoral neck fracture, a unilateral total hip arthroplasty, patients who have had a hip osteotomy in the past, and incomplete medical or radiographic records. All subjects gave their informed consent for inclusion before they participated in the study. The study was conducted in accordance with the Declaration of Helsinki, and the protocol was approved by the Ethics Committee of Opole University (UO/0004/KB/2021).

The study had been approved by the local ethics committee. Application of the inclusion and exclusion criteria ultimately resulted in 42 patients (16 females and 26 males), in the age range between $54-78$ years, being included in this retrospective study. In a group of 42 patients, 36 of them performed physical work, and 6 had office work. Eight of the patients are still economically active and 34 are currently retired. In the analyzed group, the right lower limb was dominant in 38 patients, while the left lower limb was dominant in 4 patients. The implantation procedures had been performed by one of three orthopedic surgeons, who used the same surgical technique (the same insertion and placement of the implant). All patients followed the same postoperative rehabilitation protocol.

Based on the radiographic and medical records, we analyzed the length of hospital stay, duration of intraoperative anesthesia, stem size, cup size, prosthetic head size, type of cup insert, and number of complications (prosthesis dislocation, periprosthetic fracture, infection, and delayed wound healing). 
We compared the first and second total hip arthroplasty in terms of all these evaluated parameters.

In order to establish predictive factors for the second procedure, we analyzed the correlation between the following parameters from the first-stage and second-stage procedures:

duration of hospital stay, time of anesthesia during surgery, stem size, cup size, prosthetic head size, type of cup insert, and number of complications per patient.

The obtained data were statistically analyzed using Statistica 13.1. The Shapiro-Wilk test was used to check for normality of distribution. Student's $t$-test and Pearson's $r$ correlation were used to compare the variables. The level of statistical significance was set at $p<0.05$.

\section{Results}

A total of 42 patients ( 16 females and 26 males) who had undergone two-stage bilateral total hip arthroplasty due to osteoarthritis in both hips were evaluated in this study. The results have been summarized in Tables 1 and 2. The first hip joint to be operated on was the right one in 27 cases and the left one in 15 cases.

Table 1. Characteristics of data from the first and second surgery.

\begin{tabular}{ccccc}
\hline Variable & First Operation & $\begin{array}{c}\text { Second } \\
\text { Operation }\end{array}$ & df & $p$-Value \\
\hline $\begin{array}{c}\text { Mean duration of hospital } \\
\text { stay [days] }\end{array}$ & $5.83 \pm 1.91$ & $5.4 \pm 1.46$ & 82 & $0.2522^{*}$ \\
$\begin{array}{c}\text { Mean time of anesthesia } \\
\text { during surgery [minutes] }\end{array}$ & $108.09 \pm 23.68$ & $104.52 \pm 20.77$ & 82 & $0.4646^{*}$ \\
$\quad \begin{array}{c}\text { mean stem size } \\
\text { mean cup size }\end{array}$ & $7.11 \pm 4.42$ & $7.09 \pm 4.57$ & 82 & $0.9807^{*}$ \\
Mean prosthetic head size & $0.64 \pm 3.52$ & $53.04 \pm 3.62$ & 82 & $0.6055^{*}$ \\
* Student's $t$ test; data are the mean \pm standard deviation. & & & & \\
\hline
\end{tabular}

Table 2. Correlation between data from the first and second surgery.

\begin{tabular}{ccc}
\hline Variable & Correlation Coefficient & $p$-Value \\
\hline Mean duration of hospital stay [days] & -0.0189 & $p<0.05^{*}$ \\
Mean time of anesthesia during surgery & 0.0737 & $p<0.06^{*}$ \\
[minutes] & 0.9324 & $p<0.06^{*}$ \\
Mean stem size & 0.7086 & $p<0.07^{*}$ \\
Mean cup size & 0.5121 & $p<0.08^{*}$ \\
Mean prosthetic head size &
\end{tabular}

*Pearson's r correlations.

The mean duration of hospital stay at the time of the first total hip arthroplasty was 5.83 days. This was compared with the mean duration of hospital stay at the time of the second procedure, which was 5.4 days. This difference was not statistically significant $(p=0.2522)$, (Figure 1).

The mean stem sizes used during the first and second total hip replacement procedures were 7.11 and 7.09, respectively. These figures showed no significant difference between the stem sizes used during the first and second procedure $(p=0.9807)$ (Figure 2). There was a strong correlation between the stem size from the first surgery and that used during the second surgery (correlation coefficient $=0.9324$ ) (Figure 3). 


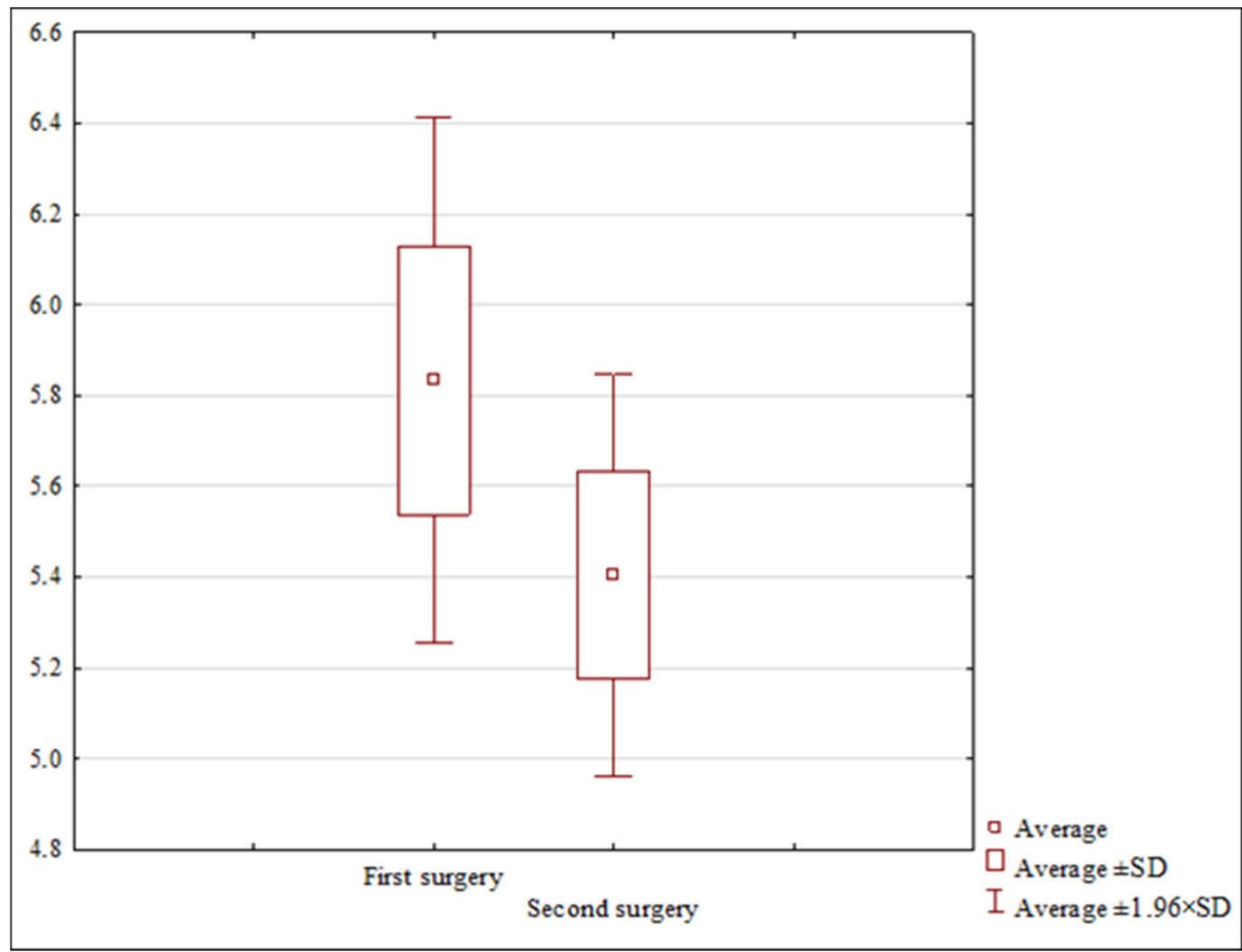

Figure 1. Duration of hospital stay [days]—first and second surgery.

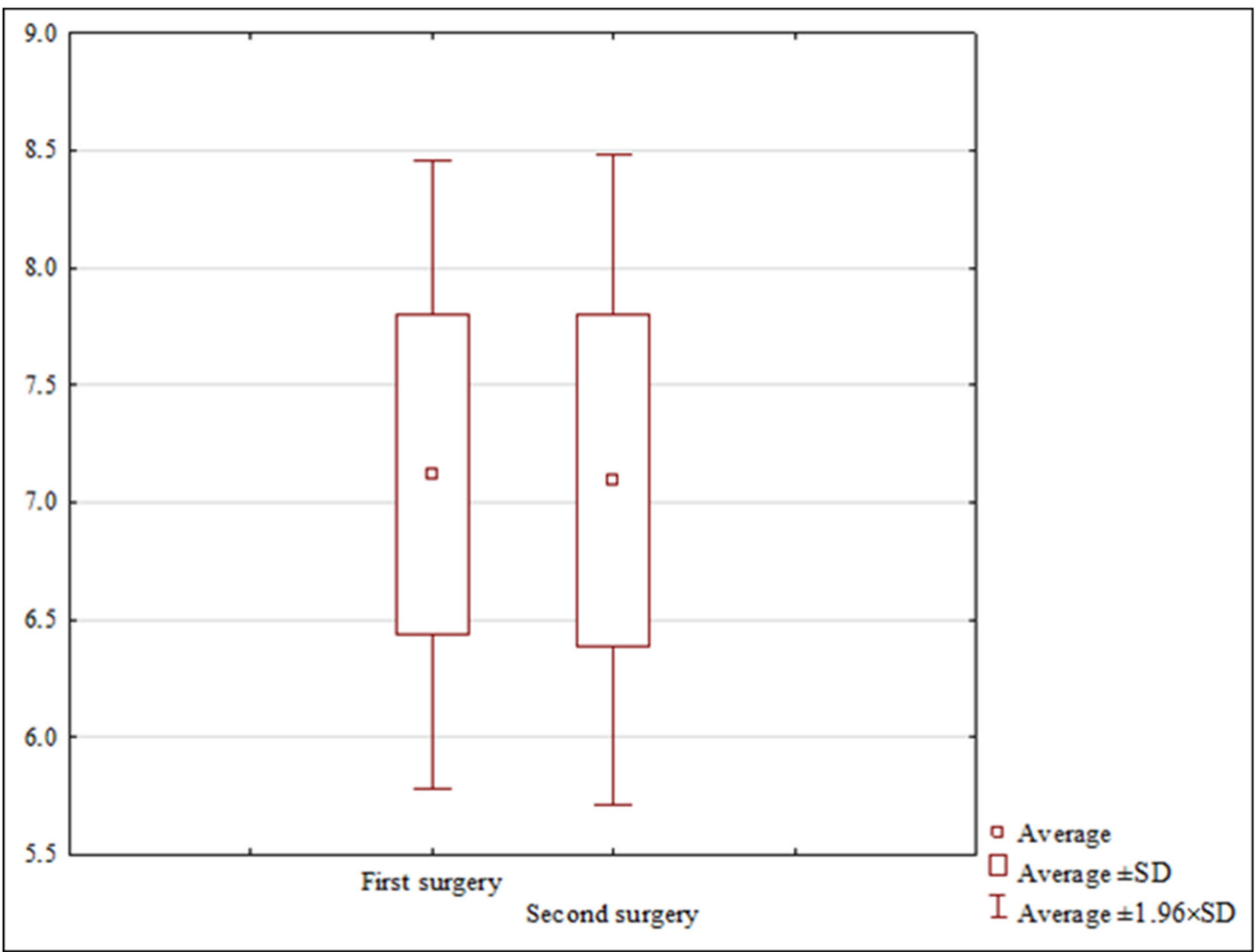

Figure 2. Stem size-first and second surgery. 


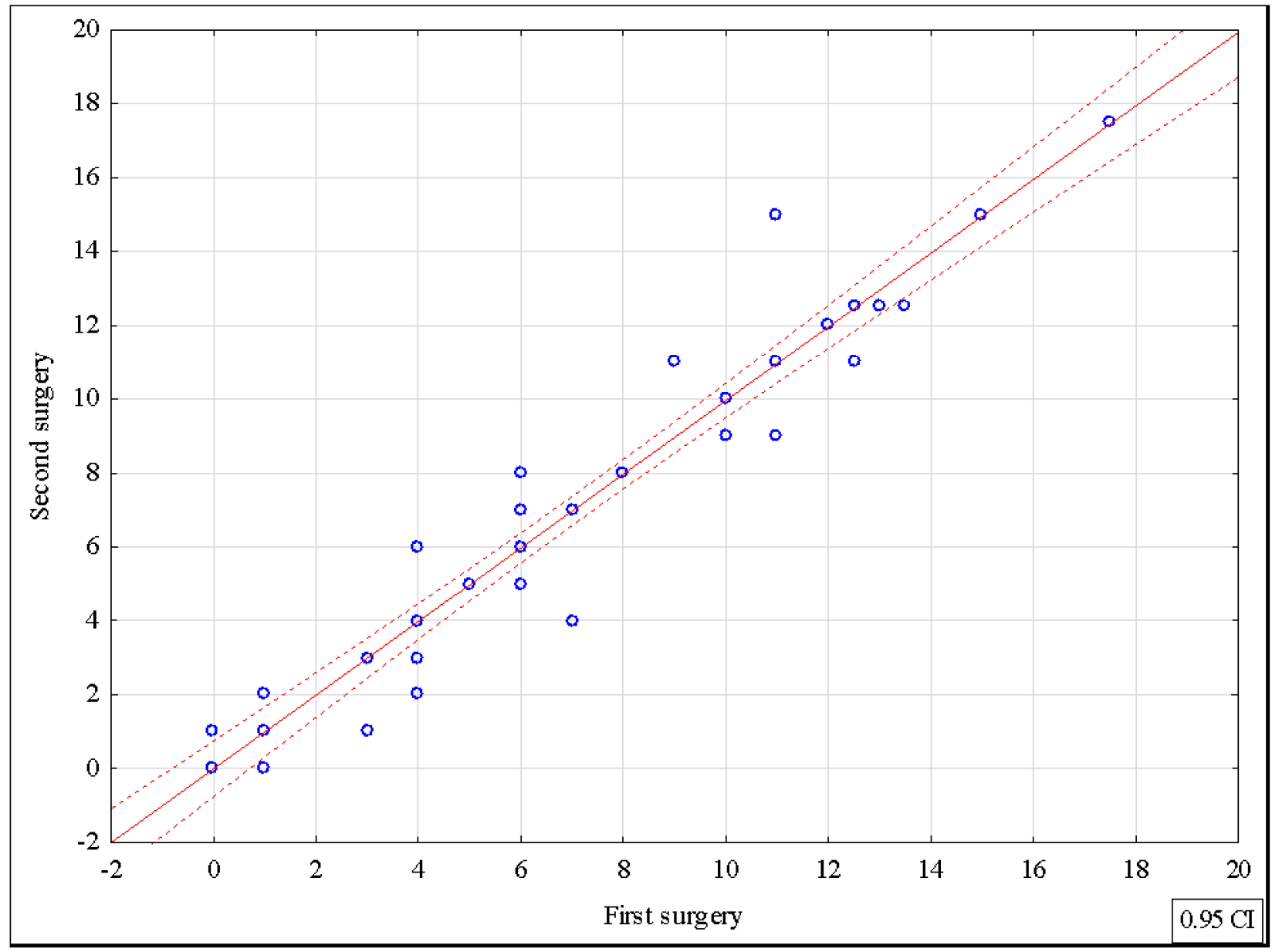

Figure 3. Correlation between stem size from the first and second surgery.

The mean sizes of endoprosthetic cups used at the first and second total hip replacement procedures were 52.64 and 53.04, respectively, which made the difference nonsignificant $(p=0.6055)$. In fact, there was a strong correlation between the cup size used during the first and that used during the second procedure (correlation coefficient $=0.7086$ ) (Figure 4).

There were no significant differences between the mean prosthetic head size at the first and second surgery ( $p=0.3334$ ). The correlation coefficient for the correlation between the prosthetic head size used at the first and second arthroplasty was 0.5121.

The cup type used during the first and second surgery showed no difference $(p=1)$.

The mean duration of anesthesia used during the first and second total hip replacement surgery was $108.09 \mathrm{~min}$ and $104.52 \mathrm{~min}$, respectively. Thus, the duration of anesthesia during the two procedures showed only a non-significant difference $(p=0.4646)$.

We recorded a mean of 0.07 complications per patient at the first surgery and 0.02 at the second surgery. A comparison between the two stages of bilateral total hip replacement showed the difference in the number of complications per patient to be non-significant $(p=0.3141)$. In four cases there was delayed wound healing (due to hematoma reabsorption); however, the wound swab cultures collected during the hospital stay of those patients were negative. In fact, there were no cases of surgical wound infection, endoprosthesis dislocation, or periprosthetic fracture either following the first or the second procedure. 


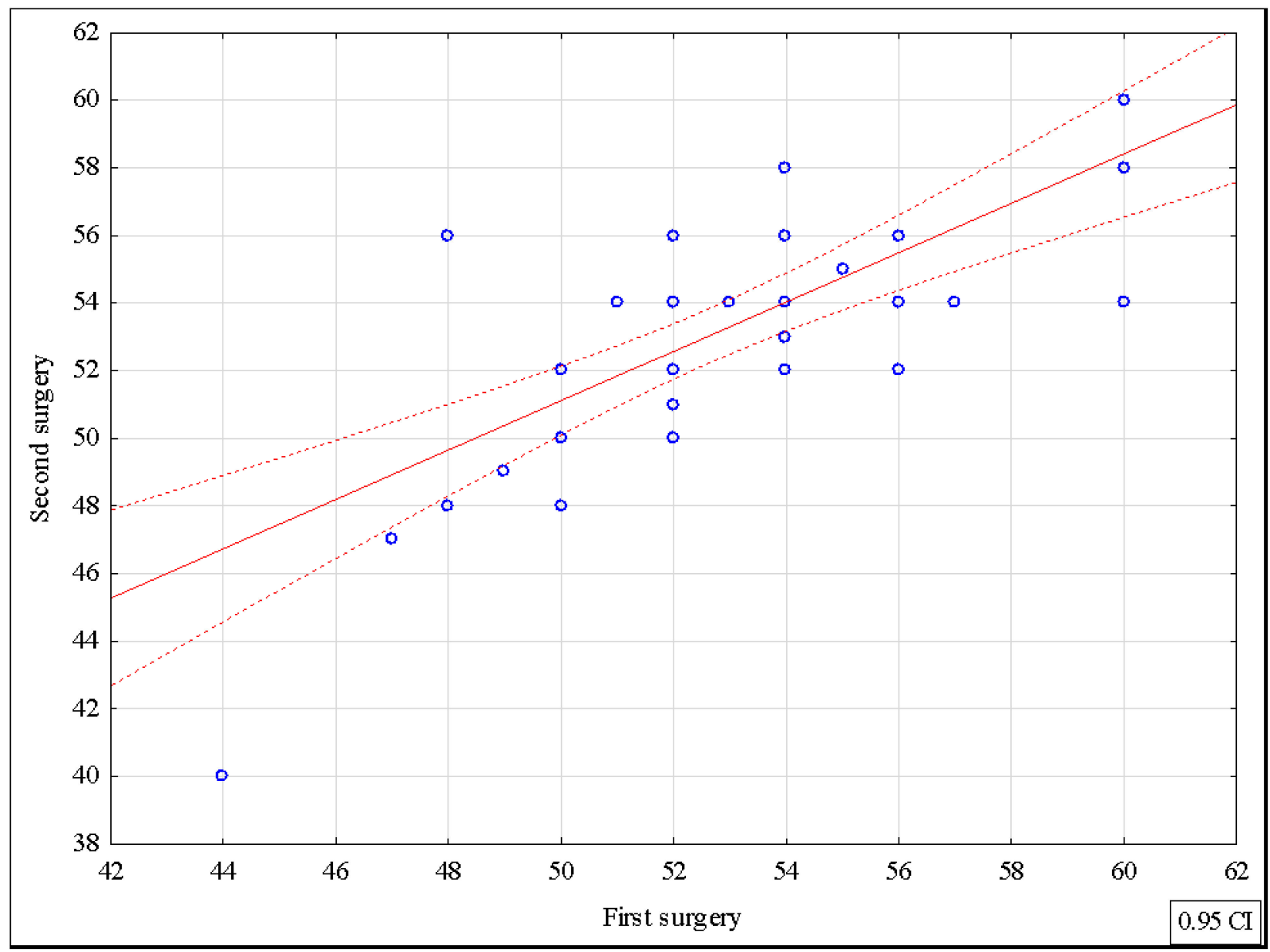

Figure 4. Correlation between cup size from the first and second surgery.

\section{Discussion}

The purpose of total hip arthroplasty is to restore the function of the treated limb, improve the range of motion, reduce pain, and allow the patient to return to their normal activities, which improves the quality of life and the state of health in all its aspects $[8,10]$. Approximately 10-25\% of people with bilateral degenerative joint disease of the hip undergo bilateral total hip replacement surgery [3,7-9,11]. There is no 'gold standard' for the management of patients with bilateral osteoarthritis of the hip [3-20]. Some orthopedic surgeons prefer a simultaneous (one-stage) bilateral total hip arthroplasty $[6,9,10,12-15,20]$, whereas others prefer a two-stage procedure, which involves operating on one hip joint at a time $[3,4,7]$.

The two-stage bilateral total hip arthroplasty may be better than the one-stage procedure in that it affords an opportunity to establish the predictive factors for the second surgery [3,4]. The issue of bilateral total hip arthroplasty has not yet been thoroughly explored, particularly the evaluation of similarities or predictive factors for the second arthroplasty [3-5]. Most authors have compared one-stage and two-stage bilateral total hip replacement procedures in terms of the number of complications [5-11,15-18]. In the case of bilateral total hip replacement procedures, it is important to predict the risk factors for the second-stage surgery [4]. Therefore, assessing patients who have undergone bilateral total hip replacement is very important, since it may help establish predictive factors for the subsequent procedure on the collateral hip, plan the second surgery, and prepare the surgeon for possible challenges and complications, which would improve the treatment process. 
Based on an analysis of 19 studies in 59,257 patients, Huang et al. observed that a simultaneous bilateral total hip arthroplasty carries an increased risk of pulmonary embolism and deep vein thrombosis than a two-stage procedure [10]. In their study involving 72 bilateral total hip replacement patients, Möllenhoff et al. reported an association between the duration of the interoperative period in two-stage total hip arthroplasty and both the survival time of the first prosthesis and mobility of the patients [3]. Those authors observed the best outcomes in the subgroup of patients with the time interval between the procedures of 1-3 years [3]; however, they did not evaluate any other predictive factors or compared the parameters of the two endoprostheses. Lampropoulou et al. observed a lack of significant differences in the survivorship rates of unilateral and bilateral total hip replacement over a long-term follow-up [4]. Neither patient sex nor age proved to have any effect on the survivorship rates of hip endoprostheses in that study [4]. Moreover, the initial diagnosis, order of the procedures, or interoperative interval also appeared not to be predictive factors and showed no statistically significant correlation with the survivorship of bilateral hip endoprostheses [4]. Lorenze et al. assessed 40 patients after a one-stage bilateral hip arthroplasty and compared them with a group of patients theoretically undergoing a two-stage procedure. An assumption was made that each unilateral case represented the first side of bilateral total hip replacement performed during two separate hospitalizations [6]. The authors reported no differences in the number of complications, procedure duration, or loss of blood; however, they observed a shorter duration of hospital stays for one-stage procedures [6].

Garland et al. reported no significant differences in postoperative mortality between the patients with one-stage and two-stage bilateral total hip arthroplasty [9].

Agarwal et al., who assessed 56 patients following a two-stage bilateral hip arthroplasty, reported the mean length of hospital stay of 5.6 days [20], which is similar to that in our observations. Our study demonstrated no significant difference between the length of hospital stay between the first and second stages of bilateral total hip replacement procedures.

The results of our study demonstrated no significant differences in the duration of anesthesia during the first and second procedures. Neither were there strong correlations between the duration of anesthesia or duration of the hospital stay for first and second procedures.

We observed a definite similarity and a strong correlation between the stem size used during the first and second surgery. Moreover, we observed a definite similarity and a strong correlation between the acetabular cup size used during the first and second procedures.

The prosthetic head size and cup insert type used in our study were symmetrical in both hips. There was also no significant difference in the mean number of complications per patient during the first and second surgery.

In our study population, there were 27 patients who had their right hip joint replaced first and 15 who had their left hip joint replaced first. Notably, the majority of patients had their right hip operated on first, which may suggest more rapid hip joint degeneration on the right side. Most of the patients we assessed performed physical work, had their right dominant leg, and were retired. The dominant right leg may have contributed to the earlier development of degenerative changes in the right hip; however, the small sample size may be a limitation.

The small sample size of 42 , evaluation of medical records only, and the retrospective nature of the study may be considered its limitations; however, some other authors have also presented retrospectively collected data $[3-6,9,11,16,17,20]$.

The strengths of our study include the fact that all procedures were performed by one of three orthopedic surgeons (with the use of the same surgical technique), and the exclusive recruitment of patients with osteoarthritis of the hip. In the future, we are planning to evaluate a larger group of bilateral total hip arthroplasty patients. In the future, we are planning more detailed research to assess the insertion and placement of the implant. 
Our study showed predictive factors and an inter-procedure similarity of the assessed parameters during the second total hip arthroplasty (on the other side). This can help surgeons plan the course of treatment, which may help lower the risk of complications, yield better outcomes, and improve patient satisfaction during the second (contralateral) total hip replacement procedure.

\section{Conclusions}

Our study results showed symmetry of the duration of anesthesia, length of hospital stay, the number of complications per patient, stem size, prosthetic head size, cup insert size, and cup insert type at the first and second surgery in patients with two-stage bilateral total hip arthroplasty.

We observed a strong correlation between the stem sizes of the first and second hip endoprostheses. There was also a strong correlation between the cup sizes used during the first and second surgery.

Author Contributions: Conceptualization, K.K. and P.M.; data curation, K.K., Ł.T. and M.C.; formal analysis, K.K. and P.M.; investigation, K.K. and P.M.; methodology, K.K., Ł.T. and P.M.; project administration, K.K. and P.M.; resources, K.K. and P.M.; software, K.K.; supervision, K.K. and P.M.; validation, K.K., Ł.T. and P.M.; visualization, K.K., Ł.T., J.W. and P.R.; writing—original draft, K.K., Ł.T., M.C., J.W., P.R. and P.M.; writing—review and editing, K.K., M.C., P.R. and P.M. All authors have read and agreed to the published version of the manuscript.

Funding: This research received no external funding.

Institutional Review Board Statement: The study was conducted according to the guidelines of the Declaration of Helsinki, and approved by the Institutional Review Board of Opole University (protocol code U) /0004/KB/2021, date of approval 17.06.2021.

Informed Consent Statement: Informed consent was obtained from all subjects involved in the study.

Data Availability Statement: Not applicable.

Conflicts of Interest: The authors declare no conflict of interest.

\section{References}

1. Kremers, H.M.; Larson, D.R.; Crowson, C.S.; Kremers, W.K.; Washington, R.E.; Steiner, C.A.; Jiranek, W.A.; Berry, D.J. Prevalence of Total Hip and Knee Replacement in the United States. J. Bone Jt. Surg. Am. Vol. 2015, 97, 1386-1397. [CrossRef] [PubMed]

2. Bedard, N.A.; Elkins, J.M.; Brown, T.S. Effect of COVID-19 on Hip and Knee Arthroplasty Surgical Volume in the United States. J. Arthroplast. 2020, 35, S45-S48. [CrossRef] [PubMed]

3. Möllenhoff, G.; Walz, M.; Muhr, G.; Rehn, J. Bilateral hip joint endoprosthesis: The time interval as a prognostic parameter? Unfallchirurg 1994, 97, 430-434. [PubMed]

4. Lampropoulou-Adamidou, K.; Macheras, G.A.; Hartofilakidis, G. Bilateral Character of Total Hip Replacement Does Not Change the Overall Survival. HIP Int. 2014, 25, 138-141. [CrossRef] [PubMed]

5. Bülow, E.; Nemes, S.; Rolfson, O. Are the First or the Second Hips of Staged Bilateral THAs More Similar to Unilateral Procedures? A Study from the Swedish Hip Arthroplasty Register. Clin. Orthop. Relat. Res. 2020, 478, 1262-1270. [CrossRef] [PubMed]

6. Lorenze, M.; Huo, M.H.; Zatorski, L.E.; Keggi, K.J. A comparison of the cost effectiveness of one-stage versus two-stage bilateral total hip replacement. Orthopedics 1998, 21, 1249-1252. [CrossRef] [PubMed]

7. Alfaro-Adrián, J.; Bayona, F.; Rech, J.; Murray, D. One- or two-stage bilateral total hip replacement. J. Arthroplast. 1999, 14, 439-445. [CrossRef]

8. Muskus, M.; Rojas, J.; Gutierrez, C.; Guio, J.; Bonilla, G.; Llinas, A. Bilateral Hip Arthroplasty: When Is It Safe to Operate the Second Hip? A Systematic Review. BioMed Res. Int. 2018, 2018, 1-10. [CrossRef]

9. Garland, A.; Rolfson, O.; Garellick, G.; Kärrholm, J.; Hailer, N.P. Early postoperative mortality after simultaneous or staged bilateral primary total hip arthroplasty: An observational register study from the Swedish Hip Arthroplasty Register. BMC Musculoskelet. Disord. 2015, 16, 77. [CrossRef]

10. Huang, L.; Xu, T.; Li, P.; Xu, Y.; Xia, L.; Zhao, Z. Comparison of mortality and complications between bilateral simultaneous and staged total hip arthroplasty. Medicine 2019, 98, e16774. [CrossRef] [PubMed]

11. Stavrakis, A.; SooHoo, N.F.; Lieberman, J.R. Bilateral Total Hip Arthroplasty has Similar Complication Rates to Unilateral Total Hip Arthroplasty. J. Artrhoplasty 2015, 30, 1211-1214. [CrossRef]

12. Heiberg, K.E.; Figved, W. Physical Functioning and Prediction of Physical Activity After Total Hip Arthroplasty: Five-Year Followup of a Randomized Controlled Trial. Arthritis Rheum. 2016, 68, 454-462. [CrossRef] [PubMed] 
13. Heiberg, K.E.; Ekeland, A.; Bruun-Olsen, V.; Mengshoel, A.M. Recovery and Prediction of Physical Functioning Outcomes During the First Year After Total Hip Arthroplasty. Arch. Phys. Med. Rehabil. 2013, 94, 1352-1359. [CrossRef] [PubMed]

14. Temporiti, F.; Zanotti, G.; Furone, R.; Loppini, M.; Molinari, S.; Zago, M.; Galli, M.; Grappiolo, G.; Gatti, R. Functional and postural recovery after bilateral or unilateral total hip arthroplasty. J. Electromyogr. Kinesiol. 2019, 48, 205-211. [CrossRef] [PubMed]

15. Taheriazam, A.; Mohseni, G.; Esmailiejah, A.A.; Safdari, F.; Abrishamkarzadeh, H. Bilateral total hip arthroplasty: One-stage versus two-stage procedure. HIP Int. 2019, 29, 141-146. [CrossRef] [PubMed]

16. Partridge, T.C.; Charity, J.A.; Sandiford, N.A.; Baker, P.N.; Reed, M.R.; Jameson, S.S. Simultaneous or Staged Bilateral Total Hip Arthroplasty? An Analysis of Complications in 14,460 Patients Using National Data. J. Arthroplast. 2020, 35, 166-171. [CrossRef] [PubMed]

17. Micicoi, G.; de Dompsure, R.B.; Micicoi, L.; Tran, L.; Carles, M.; Boileau, P.; Trojani, C. One-stage bilateral total hip arthroplasty versus unilateral total hip arthroplasty: A retrospective case-matched study. Orthop. Traumatol. Surg. Res. 2020, 106, 577-581. [CrossRef] [PubMed]

18. Kolodziej, L.; Bohatyrewicz, A.; Jurewicz, A.; Szczypiór-Piasecka, K.; Przybył, K. Simultaneous Bilateral Minimally Invasive Direct Anterior Approach Total Hip Arthroplasty with fast track Protocol. Ortop. Traumatol. Rehabil. 2020, 22, 17-25. [CrossRef]

19. Temporiti, F.; Zanotti, G.; Furone, R.; Molinari, S.; Zago, M.; Loppini, M.; Galli, M.; Grappiolo, G.; Gatti, R. Gait analysis in patients after bilateral versus unilateral total hip arthroplasty. Gait Posture 2019, 72, 46-50. [CrossRef]

20. Agarwal, S.; Gupta, G.; Sharma, R.K. Comparison between single stage and two stage bilateral total hip replacement- our results and review of literature. Acta Orthop. Belg. 2016, 82, 484-490. [PubMed] 